\title{
Successful Outcome of Cardiac Arrest Management in a Morbidly Obese Parturient Woman During Caesarean Section Delivery: A Case Report
}

\author{
Authors: \\ *Gamal A.M. Ejaimi,' Sittelnissa S. Ahmed² \\ 1. Department of Anaesthesia and Intensive Care, Taiba Hospital, Sabah Al-Salem, \\ Kuwait \\ 2. Department of Anaesthesia and Intensive, Aseer Central Hospital, Abha, Saudi \\ Arabia \\ *Correspondence to gamalejaimi@hotmail.com
}

Disclosure: $\quad$ The authors have declared no conflicts of interest.

Received: $\quad 22.11 .19$

Accepted: $\quad 03.01 .20$

Keywords: $\quad$ Aortocaval compression, cardiac arrest (CA), caesarean section (CS), morbidly obese parturient, spinal anaesthesia.

Citation:

EMJ Repro Health. 2020;DOI/10.33590/emjreprohealth/19-00040

\section{Abstract}

During a caesarean section (CS), severe hypotension following spinal anaesthesia, aortocaval compression, and morbid obesity may cause a decrease in cardiac output resulting in cardiac arrest. Cardiopulmonary resuscitation during CS is stressful for all the attending team, which mandates the importance of a high level of skill and readiness to perform perimortem CS. Reported here is a case of 36-year-old, full-term, morbidly obese parturient female who developed cardiac arrest during an emergency CS under spinal anaesthesia before delivery of the baby. Cardiopulmonary resuscitation was performed and enhanced with resuming of left lateral tilt and completion of perimortem CS. A healthy baby was delivered, and the operation was completed with good homeostasis.

\section{INTRODUCTION}

Compression of the inferior vena cava and abdominal aorta by the gravid uterus in addition to hypotension following spinal anaesthesia during caesarean section (CS) may be enhanced by morbid obesity. It may result in a severe decrease in venous return (preload) and afterload; furthermore, a decrease in cardiac output may occur. Without early recognition and correction, cardiac arrest (CA) may ensue. The positioning of the uterus and abdomen during CS is critical to avoid aortocaval compression. It is achieved by tilting of the operation table to the left side or by placing a wedge under the right buttock

of the patient. The left lateral tilt could make the operation more difficult; however, it facilitates effective cardiopulmonary resuscitation (CPR) and obstetricians should be familiar with delivering a baby in this positioning. CPR during CS is stressful for all attending staff, and mandates the importance of more skills and the ability to perform or complete a perimortem CS. ${ }^{1-3}$

\section{CASE REPORT}

A 36-year-old (gravida 3, para 2) full-term, morbidly obese parturient woman was planned for emergency CS as a result of a failure to progress after induction of labour. The patient 
was lying in the left lateral position with a size 18-gauge intravenous cannula at the dorsum of the right hand and dextrose in normal saline was running. She had a body weight of $136 \mathrm{~kg}$, height of $162 \mathrm{~cm}$, and a BMI of 51.8. She was not diabetic, hypertensive, or asthmatic, and had irregular antenatal care. The bulk of her increasing body weight was during the prior 2 years. Her last meal was approximately 3 hours before and contained milk. Vital signs were a heart rate of 107 beats $/ \mathrm{min}$, blood pressure 125/78 $\mathrm{mmHg}$, and $\mathrm{O}_{2}$ saturation 95\% (room air). No signs of fetal distress from the obstetric side were noted. A Foley catheter was in place and urine output was good. Airway assessment revealed a short neck, mouth opening of three fingers (Mallampatti Score 2), and a thyromental distance of $7 \mathrm{~cm}$. ECG was normal, and the patient's laboratory investigations were within normal range, which included full blood count, urea and electrolyte levels, and liver function test. Ranitidine $50 \mathrm{mg}$ and metoclopramide $10 \mathrm{mg}$ were administered intravenously.

She was transported to a closed operation room on a trolley in the left lateral position and wore an $\mathrm{O}_{2}$ face mask. The choice of spinal anaesthesia was discussed with the patient as the firstline procedure. Another 18-gauge cannula was inserted at the dorsum of the right hand. The patient was administered $1 \mathrm{~L}$ of intravenous normal saline and ringer lactate as preload and connected to the standard monitoring as per American Society of Anesthesiologists (ASA) guidelines. Her vital signs were a heart rate of 110 beats/min, blood pressure 128/78 $\mathrm{mmHg}$, and $\mathrm{O}_{2}$ saturation $100 \%$ ( $3 \mathrm{~L} \mathrm{O}_{2}$ face mask). Following preparation and scrubbing of the patient's back, spinal anaesthesia was administered through a long spinal needle (25 gauge) in the left lateral position with $2 \mathrm{~mL}$ of Marcaine ${ }^{\circledast}$ Heavy $0.5 \%$. The patient was positioned in a supine position with the left uterus tilted using a wedge under the right buttock. The sensory level was at T4. Vital signs were all within normal range. The abdominal preparation was achieved, and the staff discussed aortocaval compression and syndrome. Furthermore, communication was maintained between the anaesthetist and the patient during the operation. Time of uterus incision from the abdomen took approximately $6 \mathrm{~min}$ because surgical access was difficult. After uterine incision, the delivery of the baby was complicated, and 1.5 min passed without delivery of the baby.
Communication and reassurance with the patient were maintained. After manual centralisation of the uterus to facilitate the delivery of the baby, the patient became unresponsive and the radial pulse was lost. The left tilt was increased immediately, and the surgeon was notified to deliver the baby in this way because the patient had developed CA. CPR was initiated with chest compression and tracheal intubation was done with cricoid pressure. Pulse and ECG waves returned within approximately $60 \mathrm{sec}$. The baby was delivered with a total time of $2.5 \mathrm{~min}$ from uterus incision. A healthy boy of 3,900 $\mathrm{g}$ was delivered, with 1- and 5-min APGAR scores being 7 and 9, respectively. Anaesthesia was maintained with ketamine and atracurium after the patient recovered from CA with $100 \% \mathrm{O}_{2}$. The operation was finished after another 53 minutes with good homeostasis, and the estimated blood loss was $750 \mathrm{~mL}$. The patient was moved to the intensive care unit for post-CA management. She showed stable vital signs, adequate urine output, and satisfactory consciousness level. She was extubated safely one hour after the end of the operation. Her laboratory investigations were all within normal range, which included full blood count, urea and electrolyte levels, coagulation profile, and liver function test, in addition to chest X-ray and ECG. She was moved out of the intensive care unit the next morning, and discharged home on Day 5 with an uneventful postoperative stay and seen with her child 6 weeks later in the postnatal clinic in good condition. Patient consent was obtained for the publication of her case.

\section{DISCUSSION}

Although CA is a rare event during pregnancy, it represents a stressful condition as a result of the physiological modifications to the maternal physiology. Thus, resuscitation mandates specific modifications to the standard management. During CPR, a second patient's life needs to be considered in the decision-making process, although the priority is maternal life., ${ }^{1,2}$

Spinal and epidural anaesthesia might be associated with severe bradycardia or hypotension because of the prevention of reflex vasoconstriction in the blocked segment. Therefore, abrupt, severe bradycardia or sinus arrest can occur, but the outcome depends on early management. ${ }^{3}$ Compression of the inferior 
vena cava through the gravid uterus could result in reducing venous return and right atrial pressure. An acute circulatory collapse, severe enough to mimic haemorrhagic shock, in the supine position may arise and sudden bradycardia may also occur in some cases. ${ }^{4}$

Both Bezold-Jarisch reflex and amniotic fluid embolism might be a cause of CA during CS under general and spinal anaesthesia. Reflex bradycardia is a cardiovascular depression through reflex with vasodilatation and bradycardia. Neuraxial anaesthesia and general anaesthesia could induce sympathectomy, leading to a sudden vasovagal activation, resulting in extreme bradycardia and vasodilatation. Bradycardia could be treated with atropine, ephedrine, or adrenaline. At the same time, significant hypotension following spinal anaesthesia could aggravate the effect of the reflex bradycardia. ${ }^{5}$

Reduction in overweight and obesity are of great importance in leading to a decrease in morbidity and mortality. Morbid obesity no doubt complicates the pregnancy. Both pregnancy and morbid obesity have a pathophysiological change result in multiple manifestations involving different organ systems. Obesity has a deleterious effect on airway difficulty during general anaesthesia as well as haemodynamic instability during neuraxial anaesthesia. ${ }^{6}$ Morbidly obese parturient patients have a reduction in respiratory reserve and ventilatory effort. ${ }^{7}$ All these factors increase the risk for cardiopulmonary arrest ${ }^{6,7}$ and, taken together, pregnancy and severe obesity represent a complex picture and challenging issues for both anaesthetists and obstetricians. ${ }^{2,8}$

Lateral positioning during caesarean delivery is typically considered to be impractical and sometimes full lateral positioning may be essential. 7,9 Prepregnancy obesity and excessive gestational weight gain in parturient patients are associated with a high-risk pregnancy. Pregnancyinduced hypertension, CS, and greater infant size at birth are among these complications. ${ }^{10}$ Cluver et al." found that the left lateral tilt was more efficient than the right tilt, while the manual displacement was better than the left lateral tilt. They recommended large studies to support their findings. The use of tilt has a considerable role in reducing the occurrence of inferior vena cava compression during CS and labour. This can be applied effectively by using a $15^{\circ}$ tilt during CS and a $30^{\circ}$ tilt during labour. Despite this manometer, a minority of women may have susceptibility to inferior vena cava compression. ${ }^{12,13}$ The tilt of the patient will cause a significant increase in alveolararterial $\mathrm{O}_{2}$ difference and this should be treated with the administration of a high $\mathrm{O}_{2}$ concentration.

Ecker et al. ${ }^{14}$ reported CA caused by vaginal bleeding during CS in a 43-year-old multigravida woman, under spinal anaesthesia, 20 minutes after delivery of a healthy boy at 36 weeks gestation. She had chest pain, pale lips, apnoea, and rapidly became unresponsive. Successful CPR was performed, and amniotic fluid embolism was reached as the final diagnosis. CA was reported by Farrakh et al. ${ }^{15}$ in a 42-year-old, morbidly obese primigravida in the labour room during fetal blood sampling following induction of labour at term due to gestational diabetes and pre-eclampsia. CPR was performed for 3 minutes and then a perimortem CS was performed. She was transferred to theatre for suturing, and a blood transfusion was given accordingly because the total blood loss was $3.5 \mathrm{~L}$ using 1:1:1 ratio of packed red blood cells, fresh frozen plasma, and platelets. Amniotic fluid embolism was made as a clinical diagnosis. Both mother and baby showed a good outcome. ${ }^{15}$ Banaschak et al. reported a cardiac arrest in a 19-year-old woman during a laparotomy procedure for a persisting uterine haemorrhage after spontaneous delivery of a healthy baby. An air embolism complicated the manual repositioning of the uterine inversion. The autopsy showed extreme gas embolism in both arterial and venous vessels extending from the pelvis to the head. ${ }^{16}$ Recognition and initiation of CPR in the lateral position provided a good outcome for mother and baby. ${ }^{15}$ The timely perimortem CS delivery improved the chance of maternal survival. Commonly, the 'four-min rule' approach of CPR of pregnant women in the third trimester has been followed: if pulses have not returned within 4 min of the start of resuscitation, a CS should be performed within the next min. However, Benson et al. ${ }^{17}$ suggested immediate perimortem CS for better maternal and neonatal chances of survival. ${ }^{17}$ A survivor patient in one study showed a median duration from collapse to delivery of 3 min compared with 12 min among those who died. 6

Ifthereturn of spontaneouscirculation has notbeen achieved, additional interventions may include cardiopulmonary bypass and extracorporeal 
membrane oxygenation. Performing a perimortem

CS is not a part of training in obstetrics and gynaecology; however, early decision-making and continuation of the operation results in saving both lives. Knowledge gaps are significant in the science of maternal resuscitation. Team training and simulation for readiness for maternal CA should be included during training. ${ }^{15,18}$

\section{CONCLUSION}

Anaesthesia and surgery could be complicated by morbid obesity during CS. Correction of fluid status and avoidance of aortocaval compression are important. In the cases of spinal anaesthesia, all resuscitative drugs, equipment, and tools for rapid and good assistant should be ready. Furthermore, maintaining communication with the patient as well as open eyes on standard monitoring is essential. This case demonstrates that the collaboration between the anaesthesia and obstetrical team results in successful outcome management.

\section{References}

1. Montufar-Rueda C, Gei A. Cardiac arrest during pregnancy. Clin Obstet Gynecol. 2014;57(4):871-81.

2. Sia AT et al. The ongoing challenges of regional and general anaesthesia in obstetrics. Best Pract Res Clin Obstet Gynaecol. 2010;24(3):303-12.

3. Pollard JB. Cardiac arrest during spinal anesthesia: common mechanisms and strategies for prevention. Anesth Analg. 2001;92(1):252-6.

4. Kinsella SM, Tuckey JP. Perioperative bradycardia and asystole: relationship to vasovagal syncope and the Bezold-Jarisch reflex. Br J Anaesth. 2001;86(6):859-68.

5. van Liempt SW et al. Essentials in cardiac arrest during cesarean section. Clin Pract. 2015;5(1):668.

6. Mhyre JM, Bateman BT. Tipping our CAPS to the UKOSS cardiac arrest in pregnancy study. BJOG. 2017;124(9):1382.
7. Adams JP, Murphy PG. Obesity in anaesthesia and intensive care. $\mathrm{Br} \mathrm{J}$ Anaesth. 2000;85(1):91-108.

8. Bajwa SJS, Bajwa SK. Anaesthetic challenges and management during pregnancy: strategies revisited. Anesth Essays Res. 2013;7(2):160-7.

9. Campbell TA, Sanson TG. Cardiac arrest and pregnancy. J Emerg Trauma Shock. 2009;2(1):34-42.

10. Li $\mathrm{N}$ et al. Maternal prepregnancy body mass index and gestational weight gain on pregnancy outcomes. PLoS One. 2013;8(12):e82310.

11. Cluver $\mathrm{C}$ et al. Maternal position during caesarean section for preventing maternal and neonatal complications. Cochrane Database Syst Rev. 2013;3:CD007623.

12. Kinsella SM. Lateral tilt for pregnant women: why 15 degrees? Anaesthesia. 2003:58(9):835-6.

13. Kim Yl et al. Successful management of severe aortocaval compression in twin pregnancy. Obstet Gynecol. 1975;46(3):362-4.

14. Ecker JL et al. Case records of the Massachusetts General Hospital. Case 40-2012. A 43-year-old woman with cardiorespiratory arrest after a cesarean section. N Engl J Med. 2012;367(26):2528-36

15. Farrakh $\mathrm{S}$ et al. Cardiac arrest in labour leading to perimortem caesarean section in a woman with a body mass index $>46$ with a successful outcome. EMJ Repro Health. 2016;2(1):87-9.

16. Banaschak $\mathrm{S}$ et al. Fatal postpartum air embolism due to uterine inversion and atonic hemorrhage. Int J Legal Med. 2014;128(1):147-50.

17. Benson MD et al. Maternal collapse: challenging the four-minute rule. EBioMedicine. 2016:6:253-7.

18. Zelop CM et al. Cardiac arrest during pregnancy: ongoing clinical conundrum. Am J Obstet Gynecol. 2018;219(1):52-61. 